\title{
Bose-Einstein Condensates in Time-Dependent Light Potentials: Adiabatic and Nonadiabatic Behavior of Nonlinear Wave Equations
}

\author{
Y. B. Band ${ }^{1}$ and Marek Trippenbach ${ }^{1,2}$ \\ ${ }^{1}$ Department of Chemistry, Ben-Gurion University of the Negev, 84105 Beer-Sheva, Israel \\ 2 Institute of Experimental Physics, Optics Division, Warsaw University, ul. Hoża 69, Warsaw 00-681, Poland
}

\begin{abstract}
The criteria for validity of adiabaticity for nonlinear wave equations are considered within the context of atomic matter-waves tunneling from macroscopically populated optical standing-wave traps loaded from a Bose-Einstein condensate. We show that even when the optical standing wave is slowly turned on and the condensate behaves adiabatically during this turn-on, once the tunnelingtime between wells in the optical lattice becomes longer than the nonlinear time-scale, adiabaticity breaks down and a significant spatially varying phase develops across the condensate wave function from well to well. This phase drastically affects the contrast of the fringe pattern in Josephson-effect interference experiments, and the condensate coherence properties in general.
\end{abstract}

Introduction. - Recent matter-wave interference studies with Bose-Einstein condensed atoms [1] 5] have shown that one can observe phase-dependent dynamics in dilute neutral-atom systems, in complete analogy with those observed in Josephson-effect experiments [6]. In some of the experiments reported in Refs. [3, [1, the external potential (applied by optical standing waves to the initial ground-state Bose-Einstein condensate (BEC)) is slowly turned on. One might assume, based upon the Adiabatic Theorem of Quantum Mechanics [7,8], that the system should remain in an eigenstate, even though the nature of the eigenstate evolves in time. Moreover, adiabaticity criteria for nonlinear wave equations have been studied in connection with soliton dynamics in nearly integrable systems, and one expects on the basis of these studies that adiabaticity should be maintained with slow enough variation of the parameters of the system [9]. A spatially varying phase of the condensate wave function is a manifestation of non-adiabatic dynamics of a BEC, in the sense that the adiabatic eigenstate (the ground nonlinear eigenstate of the Gross-Pitaevskii equation (GPE) calculated using the potential at any instant of time) can be taken to be real (it does not have a spatially depenendent phase). Hence one concludes that, a spatially varying phase should not develop across the condensate in Refs. 3.4. when the optical potential is turned on very slowly. Here we present calculations corresponding to conditions similar to those reported in [3. 3 ] showing that the BEC remains adiabatic as the light potential is turned on very slowly, and the phase is constant across the condensate. The dynamics are indeed adiabatic despite the fact that nonlinear dynamics precludes the possibility of a superposition principle, which is used heavily in deriving the Adiabatic Theorem. We further show that, as the light potential is slowly increased in strength to the point where the wave packets in the individual optically-induced wells become strongly separated, and the time-scale for tunneling becomes long compared to the nonlinear time-scale, adiabaticity is destroyed and a large spatially varying phase develops across the wave function. This large inhomogeneous phase, obtained within a mean-field approximation, is due to the tunneling dynamics (induced by the kinetic energy of the BEC in the lattice potential) not being able to implement the equilibration of the phase across the BEC on the nonlinear time-scale.

The process of dynamic splitting of a condensate by an external time-varying potential was studied using two mode models [10] which show that the mean-field approximation based upon the GPE will not give a good description of the splitting process due to the slow rise of a potential barrier which cuts off tunneling processes. Our results show that, even within a mean-field approximation, a sudden inhomogeneous phase build-up begins to develop when the tunneling time becomes comparable to or larger than the nonlinear time-scale, and the process of optical lattice turn-on ceases to be adiabatic when this happens. A transition occurs when these two time-scales become comparable, already within a mean-field GPE approach. The spatially varying phase adversely affects the fringe contrast in interference experiments performed on the BEC wavepackets.

Adiabaticity in nonlinear systems can be studied in various regimes. Denoting $\tau_{A D}$ as the quantum-mechanical linear adiabatic time-scale determined in terms of the difference of the (linear) energy eigenvalues [7] and $\tau_{N L}$ the nonlinear time-scale [11] (see below), the simplest regime is one in which the the duration of the dynamical process being studied, $T$, satisfies the condition, $\tau_{A D} \ll T \ll \tau_{N L}$. In this case, adiabaticity is insured by the Adiabatic Theorem due to the first inequality, and nonlinearity cannot play a significant role in the dynamics due to the second inequality. Hence, the dynamics must be adiabatic. The regime in which the experiments of Refs. 3, 3 , are carried out satisfy the condition $\tau_{A D}, \tau_{N L} \ll T$, and the nonlinearity does play an important role in the dynamics. Nevertheless, as described below, the dynamics are indeed adiabatic, until the strength of the optical lattice is so large that the wave packets in the individual wells become almost completely separated. 
We consider Bose-Einstein condensed ${ }^{87} \mathrm{Rb}$ atoms in the $\left|F=2, M_{F}=2\right\rangle$ hyperfine state confined in an array of optical traps in a gravitational field. The atoms are trapped at the antinodes of a vertically oriented red-detuned optical standing wave, which are separated by $\Delta z=\lambda / 2$, where $\lambda=840 \mathrm{~nm}$ is the wavelength of light used to confine the atoms. The depth of the optical potential is proportional to the intensity of the light; the intensity is initially zero and linearly increases with time. The initial BEC is cigar shaped with $10^{4}$ atoms in a static harmonic trap potential $V_{h o}(\mathbf{r})=\frac{m \omega_{z}}{2} z^{2}+\frac{m \omega_{x, y}}{2}\left(x^{2}+y^{2}\right)$ with frequencies $\omega_{z}=2 \pi \times 19 \mathrm{~Hz}$, and $\bar{\omega}=2 \pi \times 33 \mathrm{~Hz}\left(\bar{\omega}=\left(\omega_{x} \omega_{y} \omega_{z}\right)^{1 / 3}\right.$ with $\left.\omega_{x}=\omega_{y} \equiv \omega_{x y}\right)$ 朋. The light-potential experienced by the atoms in the BEC is given by $V_{L}(z, t)=V_{0}(t)\left(1+\cos \left(2 k_{L} z\right)\right)$ where the well-depth, $V_{0}(t)=125 t\left[E_{R}\right]$, varies linearly with time. The recoil energy $E_{R}=\frac{\hbar^{2} k_{L}^{2}}{2 m}$ is the kinetic energy gained by an atom absorbing a photon from the optical lattice, where $m$ is the atomic mass, and the photon wavevector is $k_{L}=2 \pi / \lambda$. The rate of increase of the light-potential, $\left(125\left[E_{R} / s\right]\right)$, is sufficiently slow, as shown below, that the dynamics of the BEC is adiabatic for much of the turn-on. After some time, the harmonic potential and the lightpotential are switched-off (dropped) releasing the atoms to fall under the influence of gravity. In the experiments reported in Ref. [4], the atoms are held in the optical lattice for a short time (2.5 ms) after switching off the harmonic potential, allowing the gravitational potential difference between wells to affect the phase difference between wells, and absorption images are taken $8 \mathrm{~ms}$ after the optical potential is turned off and the atoms begin to free-fall.

Theoretical Formulation - The mean-field dynamics can be determined in terms of the time-dependent GPE, $i \hbar \frac{\partial \psi(\mathbf{r}, t)}{\partial t}=\left(p^{2} / 2 m+V(\mathbf{r}, t)+N_{0} U_{0}|\psi|^{2}\right) \psi$, where $V(\mathbf{r}, t)=V_{h o}(\mathbf{r})-m g z+V_{L}(z, t), U_{0}=\frac{4 \pi a_{0} \hbar^{2}}{m}$ is the atom-atom interaction strength that is proportional to the $s$-wave scattering length $a_{0}$, and $N_{0}$ is the total number of atoms. We solve the time-dependent GPE using a split-operator fast Fourier transform method to propagate an initial state of the BEC in time in the presence of the harmonic potential, gravity and the time dependent optical lattice; the initial state is determined by propagating in imaginary time with vanishing optical lattice potential [11]. Due to the large number of grid points necessary in the lattice direction $(z)$, and the large number of time steps necessary to propagate the GPE to completion of the dynamics, we found it necessary to convert the 3D GPE into an effective 1D GPE with similar dynamics. This is carried out using the following procedure. The 1D GPE is written in terms of characteristic time-scales $t_{D F}$ for diffraction and $t_{N L}$ for the nonlinear interaction, in the following manner [11, 12]:

$$
\frac{\partial \psi}{\partial t}=i\left[\frac{r_{T F}^{2}}{t_{D F}} \frac{\partial^{2}}{\partial z^{2}}-V(z, t) / \hbar-\frac{1}{t_{N L}} \frac{|\psi|^{2}}{\left|\psi_{m}\right|^{2}}\right] \psi .
$$

Here, $r_{T F}=\sqrt{2 \mu /\left(m \bar{\omega}^{2}\right)}$ is the Thomas-Fermi radius, $\mu$ is the initial chemical potential, $\mu=\frac{1}{2}\left(\frac{15 U_{0} N}{4 \pi}\right)^{2 / 5}\left(m \bar{\omega}^{2}\right)^{3 / 5}$, $t_{D F}$ is the diffraction time $t_{D F}=2 m r_{T F}^{2} / \hbar$ and $\left|\psi_{m}\right|$ is the maximum initial magnitude of the wave function defined in terms of the initial nonlinear time by $t_{N L}=(\mu / \hbar)^{-1}=\left(N U_{0}\left|\psi_{m}\right|^{2} / \hbar\right)^{-1}$ [11]. We take all lengths in the GPE (1) in units of the Thomas-Fermi radius along the $z$ axis, and we call this length unit $r_{z} \equiv r_{T F, z}$. The key to obtaining physically relevant dynamics using the 1D GPE, is to use (1) $\omega_{1 D}=\left(\frac{2 \mu}{m r_{z}}\right)^{1 / 2}$, and $(2) N_{1 D}=N_{0}\left(\bar{\omega} / \omega_{1 D}\right)^{3}$. These two equations insure that the Thomas-Fermi radius and the nonlinear time (or the chemical potential) remain as in $3 \mathrm{D}$ world. We checked to confirm that this procedure gives the same wave function as the 1D projection of the 3D GPE solution for our studies using shorter propagation times.

Numerical Results - Starting from the BEC without any optical lattice present, we begin to increase the optical potential with the linear ramp $V_{0}(t)$ mentioned earlier. Fig. 1 1 shows the magnitude and phase of the Gross-Pitaevskii wave function, $\psi(z, t)=|\psi(z, t)| \exp (i \theta(z, t))$ as a function of position in the optical lattice, $z / r_{z}$, when the well-depth of the optical lattice is 10 and $15 E_{R}$. We have taken $z=0$ to be at the minimum of the harmonic potential plus the gravitational potential, i.e., we have shifted the definition of the center of the trap to the true center of the combined harmonic plus gravitational potential. About 17 wells of the optical potential are populated during the course of the dynamics. The magnitude $|\psi(z, t)|$ remains normalized throughout the propagation; if we were to average out the oscillations in $|\psi(z, t)|$ we would obtain roughly the initial BEC $|\psi(z, 0)|$. The phase $\theta(z, t)$ is nearly spatially independent, and the magnitudes $|\psi(z, t)|$ in Fig. 1 are very nearly equal to the eigenstates of the GPE with the optical potential present at these times. The slight variation of the phases with position $z$ indicate the small degree of the nonadiabaticity that results during the dynamics. The phases $\theta(z, t)$ vary as a function of time by a spatially independent constant due to the dynamics but this ( $z$-independent) constant phase is not physically significant in the experiments of Refs. [3, 4 . The phase $\theta(z, t)$ at the time when the depth of the optical lattice is $10 E_{R}$ has been shifted up in Fig. 目 by unity so that it could be plotted conveniently.

As we increase the well-depth of the optical lattice further, a regime is reached in which the wave packets localized in the various wells become almost completely separated. When this occurs, the spatially varying phase of the wave function begins to grow significantly, and the spatially dependent variations increase as the well-depth of the optical lattice increases. Fig. 2 shows the magnitude $|\psi(z, t)|$ and phase $\theta(z, t)$ of the GP wave function as a function of position in the optical lattice, $z / r_{z}$, when the well-depth of the optical lattice is 25 and $40 E_{R}$. The magnitude of the 
wave function is almost totally within the region $z / r_{z} \in[-1.3,1.3]$. The wave packets in the optical wells are almost fully separated. The spatially dependent variations in the phase are less pronounced in the center of the trap where the variation in the peak densities of the wave packets from well to well are smaller; by $40 E_{R}$ the variation in phase across the condensate is large compared with 1 radian. The jump in the phase for $40 E_{R}$ at $z / r_{z} \approx \pm 1.1$ is artificial and due to the continuation of the inverse trigonometric function used to calculate $\theta(z, t)$ from $\psi(z, t)$. The spatial phase variation over the condensate for $50 E_{R}$ is much greater than that for $40 E_{R}$; it varies over more than $5 \pi$.

Fig. 3 shows the magnitude of the Fourier transform of the Gross-Pitaevskii wave function $\psi(k)$ versus $k$ when the well-depth of the optical lattice is 25,40 and $50 E_{R}$. The wave function has amplitude around $k=0, \pm 2 k_{L}$, although components around $k= \pm 4 k_{L}$ are also clearly visible. The width of the wavepackets increase with increasing welldepth, particularly as the spatially varying phase across the condensate becomes significant . By $50 E_{R}$, the width of Fourier components of the wave packets are a good fraction of $2 k_{L}$.

Adiabaticity is maintained throughout the course of the dynamics until the wave packets become well separated and the tunneling time becomes comparable to or larger than the nonlinear time-scale. Adiabaticity of soliton solutions of nonlinear wave equations have been extensively studied for slowly varying external conditions (for a review, see Ref. [9]). It has been shown that if the time-scale $T$ of the variation of an external parameter is slow compared the instantaneous nonlinear eigenvalue $\omega_{0}(t)$ of the nonlinear (time-independent) equation at time $t, \omega_{0}(t) T /(2 \pi) \gg 1$, the dynamics can be adiabatic. Here we see that an additional condition is required; adiabaticity breaks down when the wave packets become well separated and the tunneling time becomes longer than the nonlinear time.

It is easy to make a rough estimate of the tunneling probability from well to well using the semiclassical approximation [8],

$$
P(t)=\exp \left(-\int_{0}^{\pi /\left(2 k_{L}\right)} k(z, t) d z\right) \approx \exp \left(-\sqrt{\frac{2 m}{\hbar^{2}}} \int_{0}^{\pi /\left(2 k_{L}\right)}\left(V_{L}(z, t)\right)^{1 / 2} d z\right)=\exp \left(-2 \sqrt{4 m V_{0}(t)} /\left(\hbar k_{L}\right)\right) .
$$

The tunneling rate is then given by $R(t)=\omega_{v}(t) \times P(t)$, where the vibrational frequency $\omega_{v}(t)$ can be approximated by $\omega_{v}(t) \approx \sqrt{4 k_{L}^{2} V_{0}(t) / m}$, since the expansion of the optical potential about a minimum in the potential yields $V_{L}(z, t) \approx(m / 2)\left(\omega_{v}(t)\right)^{2} z^{2}=(m / 2)\left(4 k_{L}^{2} V_{0}(t) / m\right) z^{2}$. Hence, the time-dependent tunneling rate is $R(t)=\sqrt{8\left(V_{0}(t) / E_{R}\right)} \exp \left(-\sqrt{8\left(V_{0}(t) / E_{R}\right)}\right)\left[E_{R} / \hbar\right]$. The tunneling rate $R(t)$ can be compared with the timedependent inverse nonlinear time-scale, $\left(t_{N L}(t)\right)^{-1}=N U_{0}\left|\psi_{m}(t)\right|^{2} / \hbar=\mu(t) / \hbar$ and the time-dependent diffraction time-scale, $\left(t_{D F}(t)\right)^{-1}=\frac{\hbar\left(\omega_{v}(t)\right)^{2}}{4 \mu(t)}$. Once the tunneling time, $R^{-1}$, becomes long compared to $t_{N L}$ (and $t_{D F}$, which is typically the smallest of these time-scales), a spatially independent phase can not be maintained across the condensate by the action of the kinetic energy operator, and a spatially dependent phase develops. In our calculations, this happens beyond a lattice well-depth of about $35 E_{R}$.

If we take our calculated wave packets (shown in Figs. 1 and 2) and propagate for an additional $2.5 \mathrm{~ms}$ upon switching off the magnetic field, but leaving the optical lattice potential in place, as described in Ref. [4], our wave packets hardly change. However, upon switching off the optical potential and propagating the wave packets for 8 ms, the wave packets spread by diffusion very significantly as they fall in gravity. The resulting wave packets are considerably different upon using the 25 and $50 E_{R}$ results. The additional Fourier components of the wave packet in the $50 E_{R}$ case significantly wash out interference patterns in the density profile for this case, consistent with the measurement in [4]. Note that we are not asserting that squeezing is absent in the experiments of Ref. [4]; rather, that an improved model of the above-the-mean field effects that accounts for the spatially varying phase is necessary to quantitatively compare with the experiments.

Conclusions - We have seen that the spatially varying phase of the solution to the Gross-Pitaevskii equation becomes large when the tunneling time between wells in the optical lattice becomes comparable to or larger than the nonlinear time-scale. Adiabaticity then breaks down and the instantaneous nonlinear eigenvectors to the timeindependent Gross-Pitaevskii equation do not have the character of the dynamical solution. This nonadiabaticity takes place even when the variation of the optical potential is sufficiently slow that adiabaticity is otherwise assured. It remains to determine the above-mean-field corrections to this picture using an approach that incorporates an accurate form for the spatially varying complex order parameter.

Finally, we note that these results have implications regarding the loading of an optical lattice with atoms for quantum computing, using a Bose-Einstein condensate (BEC) source and laser beams that are slowly turned on [13]. From our studies we conclude that the laser fields must be controlled so that the density of the atoms is sufficiently low (the nonlinear term must be negligible) before tunneling between the wells is cut off by the full depth of the optical potential. Otherwise, phase variations from well to well will deleteriously affect the resulting optical lattice state. 
Useful conversations with Boris Malomed and Paul Julienne are gratefully acknowledged. This work was supported in part by grants from the U.S.-Israel Binational Science Foundation (grant No. 98-421), Jerusalem, Israel, the Israel Science Foundation (grant No. 212/01), the Israel MOD Research and Technology Unit and the Polish KBN 2/P03/B07819

[1] M. R. Andrews et al., Science 275, 637 (1997).

[2] E. W. Hagley, et al., Phys. Rev. Lett. 83, 3112 (1999); M. Trippenbach, et al., J. Phys. B 33, 47-54 (2000).

[3] B. P. Anderson and M. A. Kasevich, Science 282, 1686 (1998).

[4] C. Orzel et al., Science 291, 2386 (2001).

[5] F. S. Cataliotti et al., Science 293, 843 (2001).

[6] A. Barone and G. Paterno, Physics and Applications of the Josephson Effect (Wiley, New York, 1982).

[7] A. Messiah, Quantum Mechanics, Vol. II, Chp. 17, (N. Holland, Amsterdam, 1975).

[8] C. Cohen-Tannoudji, B. Diu and F. Laloë, Quantum Mechanics, (John Wiley, NY, 1977).

[9] Y.S. Kivshar and B.A. Malomed, Rev. Mod. Phys. 61, 763 (1989).

[10] C. Menotti, J.R. Anglin, J.I. Cirac and P. Zoller, Phys. Rev. A63, 023601, 2001; A. J. Leggett and F. Sols, Phys. Rev. Lett. 81, 1344 (1998); R.W. Spekkens and J.E. Sipe, Phys. Rev. A 59, 3868 (1999); J. Javanainen and M.Yu. Ivanov, Phys. Rev. A 60, 2351 (1999); A. Vardi and J.R. Anglin, Phys. Rev. Lett. 86, 568 (2001).

[11] M. Trippenbach, Y. B. Band, and P. S. Julienne, Phys. Rev. A62, 023608 (2000).

[12] M. Trippenbach, Y. B. Band, and P. S. Julienne, Optics Express 3, 530 (1998).

[13] G.K. Brennen, C.M. Caves, P.S. Jessen, and I.H. Deutsch, Phys. Rev. Lett. 82, 1060 (1999); D. Jaksch, et al., Phys. Rev. Lett. 82, 1975 (1999).

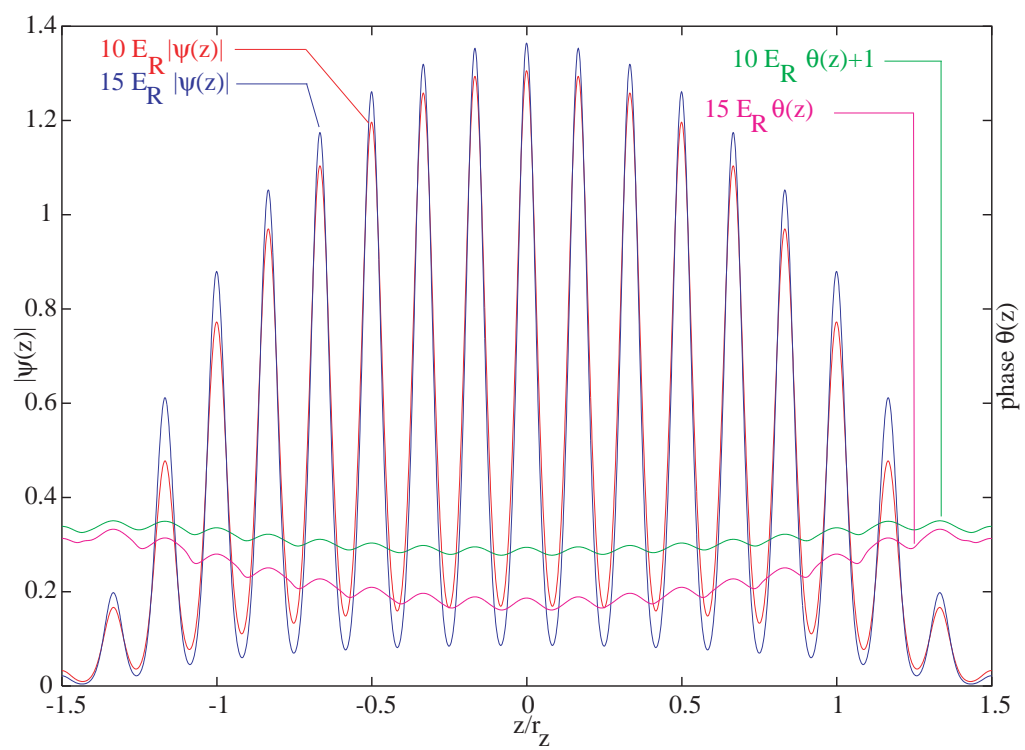

FIG. 1. Magnitude and phase of the Gross-Pitaevskii wave function as a function of position in the optical lattice. The wave function is shown when the well-depth of the optical lattice are 10 and $15 E_{R}$. 


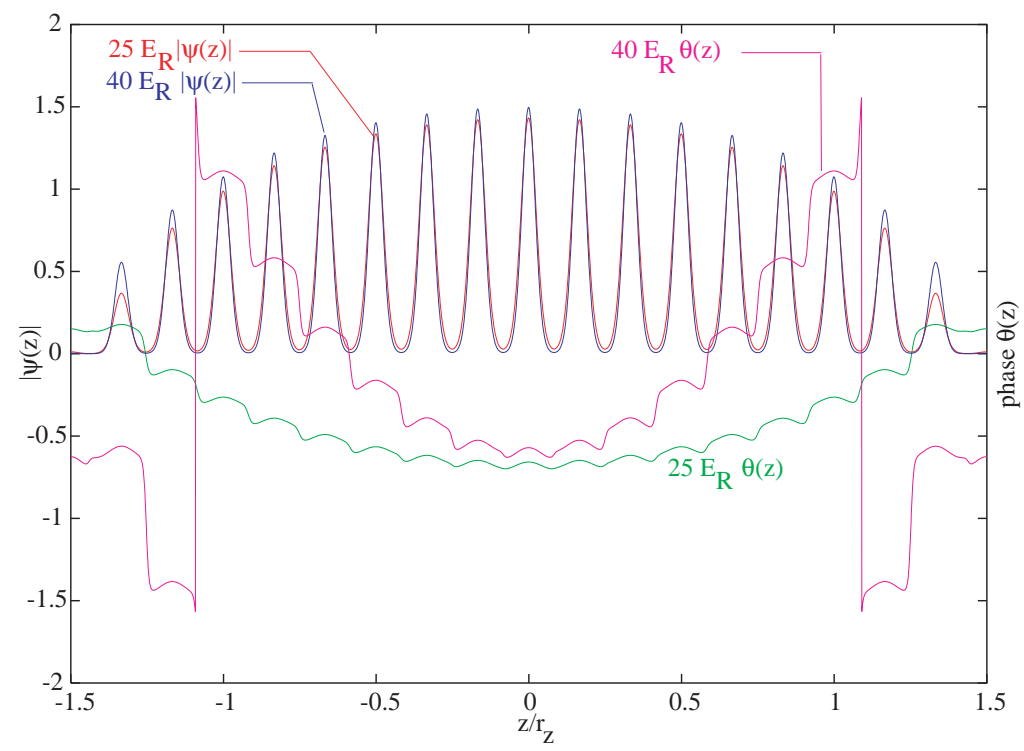

FIG. 2. Magnitude and phase of the Gross-Pitaevskii wave function as a function of position in the optical lattice. The wave function is shown when the well-depth of the optical lattice are 25 and $40 E_{R}$.

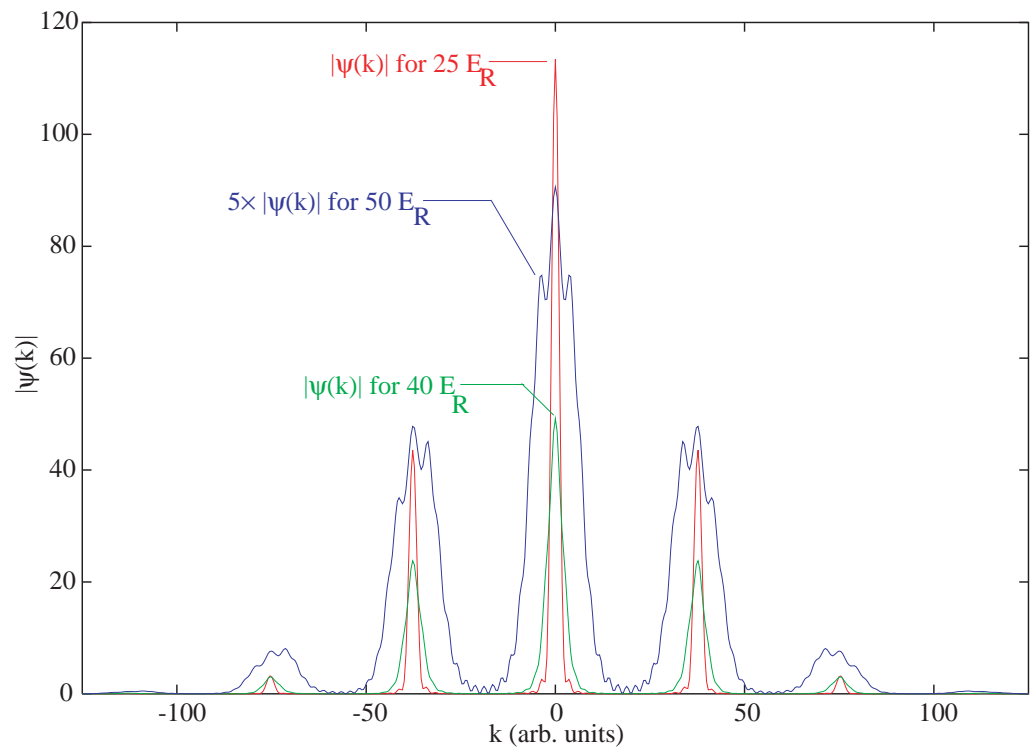

FIG. 3. Gross-Pitaevskii wave function in momentum space, $\psi(k)$ versus $k$ when the well-depth of the optical lattice are 25 , 40 and $50 E_{R}$. 